\title{
Compact modeling of Optically-Gated Carbon NanoTube Field Effect Transistor
}

\author{
Si-Yu Liao, Cristell Maneux, Vincent Pouget, Sébastien Frégonèse, and Thomas Zimmer \\ Université Bordeaux, CNRS, UMR 5218, 351 Crs de la Libération, TALENCE, France
}

siyu.liao@ims-bordeaux.fr

\section{Background}

Carbon Nanotube Field Effect Transistors (CNTFETs) have high charge sensitivity at room temperature [1]. By using this sensitivity, some nonvolatile memory devices have been demonstrated with charge trapping in $\mathrm{SiO}_{2}$ gate insulator [2, 3]. Besides, a new design of synapse-like circuit requires a multi-level nonvolatile memory [4]. For this application, and according to its high charge sensitivity, Optically-Gated Carbon Nanotube Field Effect Transistor (OG-CNTFET) appears as a good candidate thanks to optical writing and electrical erasing abilities both with single and multiple drain current levels [5, 6].

By coating a thin layer of photosensitive polymer such as poly3-octylthiophene-2,5-diyl (P3OT) over the nanotube, a CNTFET becomes an OG-CNTFET [5], as presented in Fig. 1a. Compared to the conventional CNTFET, the OG-CNTFET reveals a significant increase of the drain current below the threshold gate bias voltage. If this device is under significant powerful illumination, the gate bias will no longer control the conductivity of the CNT channel, and the optical gate will dominate the functionality. This property of variable conductance is of particular interest for neural network designs to define a third logic level. In this work, we present a compact model for OG-CNTFET. Indeed, compact modeling (i.e. SPICE-like) is a key issue for predicting the ultimate performances of these novel nano-devices in a circuit environment using standard simulation tools.

\section{Electron trapping/releasing mechanism}

When a thin film of P3OT coats on a CNTFET, this polymer acts as a p-type doping under no illumination condition [5]. We suppose that the CNT channel is electrostatically doped due to negative charges trapped at the polymer/SiO${ }_{2}$ interface in the nanotube vicinity [1].

When an OG-CNTFET is under illumination with a wavelength that can be absorbed by the polymer, electron-hole pairs are generated in the P3OT layer, and a minor part of them can be separated [7]. This polymer is known for trapping only electrons [8]. If the gate is biased positively, these trapped electrons can be homogenously attracted by the electrostatic field to the $\mathrm{P} 3 \mathrm{OT} / \mathrm{SiO}_{2}$ interface. The resulting charge density contributes to modulate the channel conductivity by screening the back gate bias. Fig. 1b describes this so-called optical gating effect. When the light is turned off, the trapped electrons are not released immediately; they are hold for about ten hours [5, 7].

The optical gating effect can be removed more rapidly by applying a negative gate voltage or a positive drain-source one [5-6, 9]. The negative gate bias provides an electrostatic field that contributes to release the trapped electrons at the $\mathrm{P} 3 \mathrm{OT} / \mathrm{SiO}_{2}$ interface. Concerning the effect of the positive $V_{\mathrm{ds}}$, we assume that it creates an electric field close to the drain electrode that contributes to sweep away the trapped carriers according to the field magnitude distribution as a function of the distance to the drain electrode (c.f. Fig. 1c).

\section{Modeling of OG-CNTFET}

Since the OG-CNTFET is a modification of the conventional CNTFET, we developed the compact model of OG-CNTFET based on the one of CNTFET. In previous works, we have presented a physics-based CNTFET electrical model [10]. It computes the relationship between the electrostatic capacitances $\mathrm{C}_{\mathrm{OX}}, \mathrm{C}_{\mathrm{SE}}$, and $\mathrm{C}_{\mathrm{DE}}$, the quantum charges $\mathrm{Q}_{\mathrm{S}}$ and $\mathrm{Q}_{\mathrm{D}}$ and the intrinsic channel potential $\mathrm{V}_{\mathrm{CNT}}$ on one hand, and the channel charge density on the other hand. The OG-CNTFET model takes into account the additional electrostatic capacitances $\mathrm{C}_{\mathrm{SO}}$ and $\mathrm{C}_{\mathrm{DO}}$ and the doping charges due to P3OT coating. When a laser spot illuminates the device, the intrinsic channel potential $\mathrm{V}_{\mathrm{CNT}}$ is directly affected by the trapping of electrons represented as a laser-power-controlled current source charging a pair of trap capacitors: $\mathrm{C}_{\text {Optic1 }}$ and $\mathrm{C}_{\mathrm{Optic2}}$ (c.f. Fig 2). These capacitors hold trapped electrons when the illumination stops. The natural recombination mechanism of traps has been taken account in this current source $\mathrm{I}_{\text {Optic. }}$ The release of the optical gating effect via $V_{d s}$ is achieved by commanding the variable resistor $R_{V d s}$. When $V_{d s}$ is negative, this resistor is infinite, whereas for positive $V_{d s}$ bias, $R_{V d s}$ becomes a finite value which depends on the amplitude of $\mathrm{V}_{\mathrm{ds}}$.

\section{Simulation results}

The calculation of internal potential $V_{\mathrm{CNT}}$ is self-consistent through Kirchoff's laws. The DC simulation results present good agreement with the measurements in the dark of the $I_{d}-V_{g s}$ characteristics of a single CNTFET and the same one with coated P3OT, as shown in Fig. 3a. 
The model also gives a good estimation of the gate bias voltage influence after laser illumination for positive values. Fig. 3b presents typical transient simulation results. Our model clearly exhibits optical writing and electrical erasing dynamics via $V_{\mathrm{ds}}$ bias that are close to experimental results presented in [5] and [9].

Hence, the model results present good agreement with previously published experimental results. Future work will include specific experiments to get accurate values of the model physical parameters such as the optical gate capacitance.

\section{References:}

[1] H. B. Peng et al., Appl. Phys. Lett. 89, (2006), 243502.

[2] M. S. Fuhrer et al., Nano Letters, 2(7), (2002), 755-759.

[3] M. Radosavljevi et al., Nano Letters, 2(7), (2002), 761-764.

[4] M. He et al., IEEE Electronics Letters, vol. 44, issue 9, (2008), 575-576.

[5] J. Borghetti et al., Adv. Mater. 18, (2006), 2535-2540.

[6] J.P. Bourgoin et al. Electron Devices Meeting, IEDM '06. International, (2006), 11-13.

[7] C. Anghel et al., Nano Letter, 8 (11), (2008), 3619-3625.

[8] C. D. Dimitrakopoulos, P. R. Malenfant, Adv. Mater. 14, (2002), 99-117.

[9] G. Agnus et al., Nano Letter, submitted.

[10] S. Fregonese et al., IEEE Transaction on Electron Devices, vol. 55, issue 6, 2008, 1317-1326.

\section{Figures:}
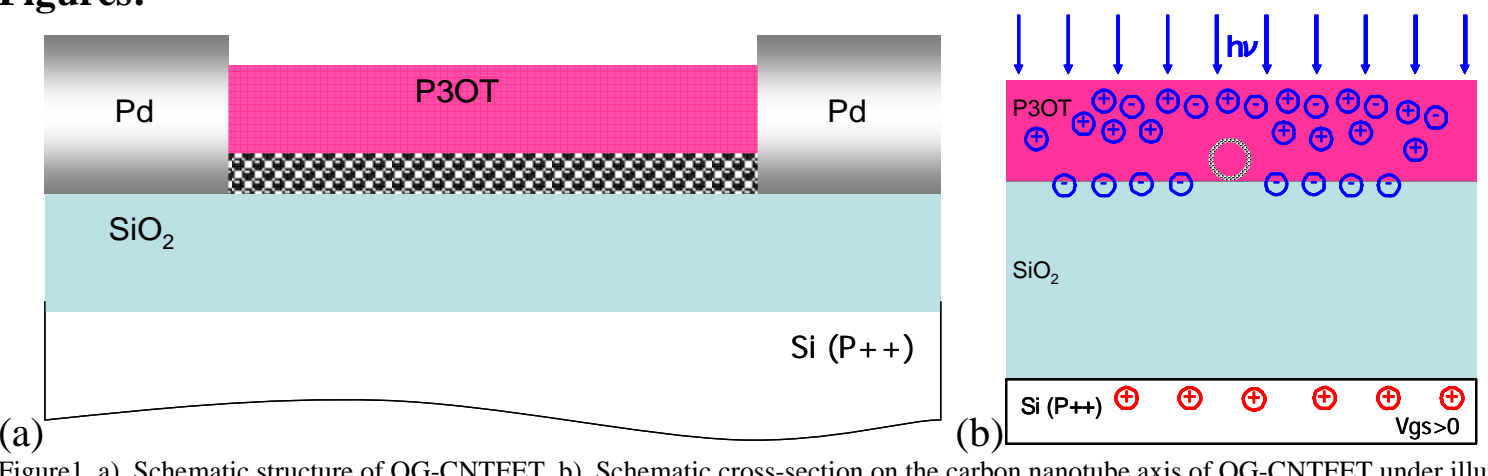

Figure1. a). Schematic structure of OG-CNTFET. b). Schematic cross-section on the carbon nanotube axis of OG-CNTFET under illumination.

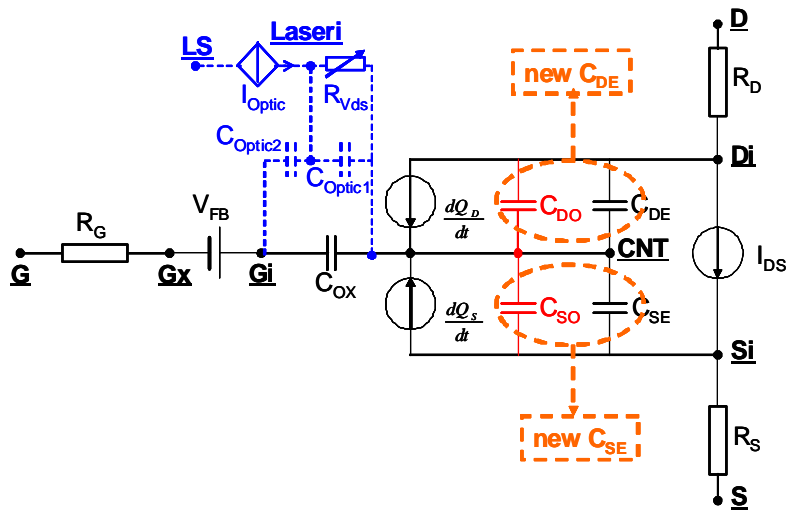

Figure 2. Electric equivalent circuit of OG-CNTFET. In black, theconventional CNTFET. In red, the P3OT influence on electrostatic capacitors. In blue, the photo-charging and discharging sub-circuit.

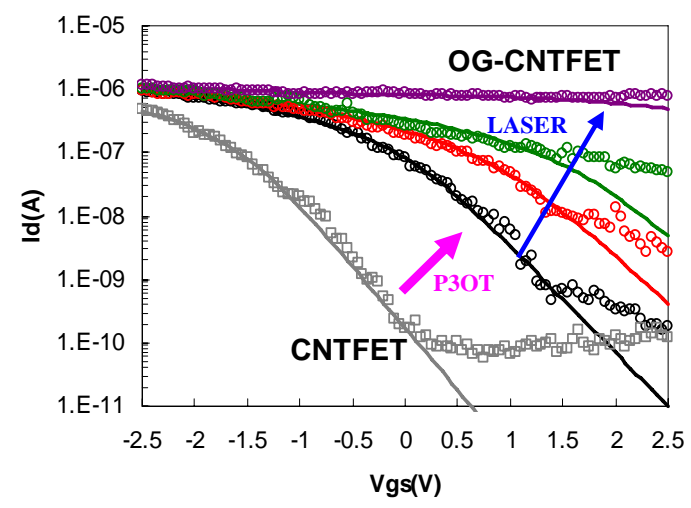

a)

b)

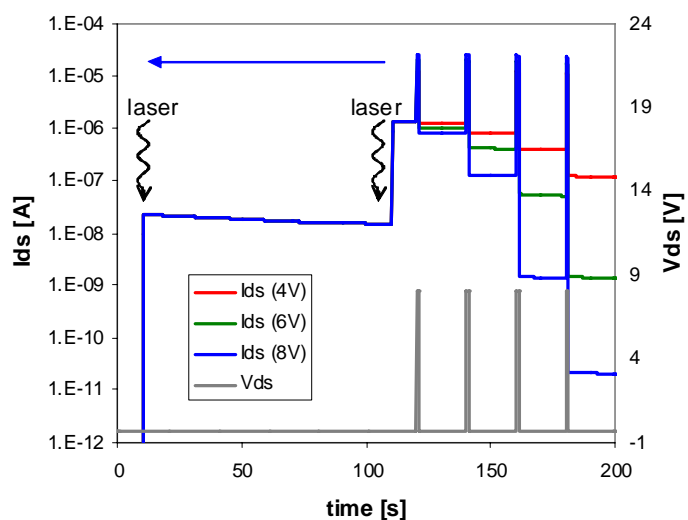

Figure 3. Simulation results. a). $\mathrm{I}_{\mathrm{d}}-\mathrm{V}_{\mathrm{gs}}$ transfer charateristics. The solid lines are simulation results, and the symble are measurements in ref. 5 . The gray curves are CNTFET in dark; the black ones are OG-CNTFET in dark; the red ones are OG-CNTFET under laser of 6nW in the spot $(2 \mu \mathrm{m})$; the green ones are under laser of $60 \mathrm{nW}$; and the purple ones are under laser of $6 \mu \mathrm{W}$. b). Transient simulation results of optical writing and eletrical erasing operations. The drain current $\mathrm{I}_{\mathrm{d}}$ is controlled by pulsed $\mathrm{V}_{\mathrm{ds}}$ of $4 \mathrm{~V}$ (red curve), of $6 \mathrm{~V}$ (green curve) and of $8 \mathrm{~V}$ (blue curve). The gray curve is $\mathrm{V}_{\mathrm{ds}}$ command. Each laser pulse is $150 \mathrm{~ms}$ long, each Vds one is $1 \mathrm{~s}$ long. 